\title{
Healthy ageing, but what is health?
}

\author{
Suresh I. S. Rattan
}

(C) Springer Science+Business Media Dordrecht 2013

\begin{abstract}
Ageing occurs in spite of complex pathways of maintenance and repair. There is no "enemy within", which has the specific evolution-selected function to cause ageing and death. This understanding of ageing should transform our approach towards interventions from therapeutic "anti-ageing" to maintaining health. But what is health? Ideally, health is a state of complete physical and mental independence in activities of daily living. But in pragmatic terms, health is a state of adequate physical and mental independence in activities of daily living. In order to identify a set of measurable, evidence-based and demonstratable parameters of health, robustness and resilience at various levels, the concept of homeodynamic space can be a useful one. Age-related health problems for which there are no clear-cut causative agents, except the complex process of ageing, may be better tackled by focusing on health mechanisms and their maintenance, rather than disease management and treatment. Continuing the disease-oriented research approaches are economically, socially and psychologically unsustainable as compared with health-oriented and preventive strategies, such as hormesis. Supporting health-oriented research is the urgency of our time.
\end{abstract}

S. I. S. Rattan ( $\square)$

Laboratory of Cellular Ageing, Department of Molecular

Biology and Genetics, University of Aarhus, Gustav

Wieds Vej 10, 8000 Aarhus C, Denmark

e-mail: rattan@mb.au.dk
Keywords Longevity - Stress - Homeostasis · Homeodynamics $\cdot$ Hormetics $\cdot$ Hormetin

We want to maintain or even enhance health; and we wish to achieve healthy ageing. But what exactly is health? Even the WHO's definition of health as "a state of complete physical, mental and social well-being and not merely the absence of disease or infirmity" (http://www.who.int/topics/mental_health/en/) is idealistic, incomplete, vague, and does not clarify what is meant by "well-being" in definitive terms. Furthermore, although everyone talks about health-there are ministries, faculties and departments of health-most of the biomedical research is dominated and supported by disease-directed thinking. This gives little understanding as to what is health, what determines health and how one maintains health and avoids the emergence of diseases in the first place. Therefore, a major change in thinking, approach and strategy is required to understand health, maintain health, improve the quality of life, extend the health-span and enhance public- and social-health (Contrada and Baum 2011; Farrelly 2012; Shelton 2013). A lack of scholarly, serious and interdisciplinary health discussion has allowed the mushrooming of numerous self-proclaimed health-specialists and health-gurus, which has created a lot of confusion, muddled thinking, and irrational, impractical and even harmful interventional strategies (Holliday 2009; Le Bourg 2013). 
If ageing is understood as an emergent phenotype due to the failure of homeodynamics, and not due to the action of any specific harmful and death-causing mechanisms (Rattan 2012b), it transforms our approach towards ageing interventions from "antiageing" to "healthy ageing". Ageing occurs in spite of the presence of complex pathways of maintenance, repair and defence, and there is no "enemy within". This viewpoint makes modulation of ageing different from the treatment of one or more age-related diseases. Another important implication of understanding ageing as the inefficiency and imperfections of homeodynamics is that the prospect of developing anti-ageing magic bullets must be abandoned. This also means abandoning enemy-oriented rhetoric, such as the "war against ageing", "defeating ageing", and "conquering ageing" etc. Instead, interventions in ageing require a "friend-oriented" approach and the use of a positive language such as maintaining health, achieving healthy ageing, successful ageing, and preserving the homeodynamics. As a biomedical issue, the biological process of ageing underlies all major diseases, and although the optimal treatment of every disease, irrespective of age, is a social and moral imperative, preventing the onset of age-related diseases by intervening in the basic process of ageing is the best approach for achieving healthy ageing and extending the health-span.

\section{Health, homeodynamics and ageing}

Health and survival of an organism is a dynamic tug between the occurrence of damage and the processes of maintenance and repair (Holliday 2007; Rattan 2012b). The traditional conceptual model to describe this characteristic has been the so-called homeostasis. However, deeper understanding of the processes of biological growth, development, maturation, reproduction and ageing have led to the realization that the term homeostasis, which means "stability through constancy", fails to incorporate dynamic themes and processes, including interacting networks and complexity in biological systems. Therefore, the term homeodynamics (Yates 1994) has replaced homeostasis to account for the fact that the internal milieu of complex biological systems is not permanently fixed, is not at equilibrium, and is a dynamic regulation and interaction among various levels of organization. All these processes and their interactions give rise to a homeodynamic space, which may also be considered as the "survival ability" or the "buffering capacity" of a biological system, and is the ultimate determinant of an individual's health, and the ability to survive and maintain a healthy state (Carnes 2011; Rattan 2007a; Rattan 2012b).

All apparently normal organisms are born with a certain extent of the homeodynamic space, but with a significant zone of vulnerability in early stages of life. The homeodynamic space undergoes expansion during growth, development and maturation, with a concurrent decrease in the vulnerability zone. The extent of homeodynamic space achieved by an individual depends both on genetic factors including polymorphisms, and on pre-natal and early-life epigenetic factors, including nutrition, infections, mental stimulation and physical activity. At the species level, biological evolutionary processes have optimised homeodynamics only for a limited period of time termed as the essential lifespan (ELS) (Carnes 2011; Rattan 2007b; Rattan 2012b), which is required for successful reproduction. However, survival beyond ELS is accompanied by a progressive shrinkage of the homeodynamic space, which is the basis of frailty, ageing, age-related diseases and eventual death. The main three characteristics of the homeodynamic space are: (1) damage control; (2) stress response (SR); and (3) constant remodelling and adaptation. Importantly, these three characteristics of health and homeodynamics can be studied at the level of biology, psychology and sociology.

Ageing is the progressive shrinkage of the homeodynamic space (Rattan 2012b). At the biological level, survival of an organism is a constant struggle between the occurrence of biochemical damage and the mechanisms of maintenance and repair. A large number of molecular, cellular and physiological pathways and their networks determine survival and longevity (Budovsky et al. 2007; Tacutu et al. 2010). At the molecular level, the theories of the mechanisms of ageing are mostly centered on the accumulation of molecular damage (Rattan 2006; Rattan 2008b). Recently some other views, such as a continuous and unregulated growth leading to a kind of quasi-programme of ageing have also been put forward (Blagosklonny 2012), which include the molecular and metabolic imbalance as the basis of ageing. 
The two other components of the homeodynamic space are the SR (Demirovic and Rattan 2013; Rattan 2012b), and constant remodelling and adaptation (Franceschi et al. 2000). All age-related changes, such as reduced hormone levels and remodelled immune profiles, may not be harmful, and may be a sign of adaptation. Similarly, the consequences of stress can be either harmful or beneficial depending on the intensity, duration and frequency of the stress, and on the price paid in terms of energy utilisation and other metabolic disturbances. But the most important aspect of SR is that it is not monotonic with respect to the dose of the stressor, rather it is almost always characterized by a nonlinear biphasic relationship, termed hormesis (Calabrese et al. 2007). Hormesis in ageing is defined as the life supporting beneficial effects resulting from the cellular responses to single or multiple rounds of mild stress (Rattan 2004; Rattan 2008a; Rattan 2008c; Rattan and Demirovic 2009). Exercise, heat, radiation, and several components of the food and spices, including flavonoids, polyphenols, and other micronutrients, have been shown to be hormesis-inducing agents, termed hormetins (Rattan 2012a).

\section{Subjective and objective measures of health}

Health is often described either in the context of the absence of one or more diseases or as a vague concept of well-being, without having any objective measures for that. Although various parameters of frailty have been proposed (Fulop et al. 2010; Hubbard and Woodhouse 2010; Mitnitski et al. 2013; Montesanto et al. 2010), direct measures of health largely remain undefined. Since one of the crucial aspects of health is the functionality of the living system, health could be defined as a state of complete physical and mental independence in activities of daily living; but this is an idealized state, which perhaps no one can have. Therefore, being healthy, in practical terms, means having ADEQUATE physical and mental independence in activities of daily living; and this state may vary widely but can be established objectively. However, the so-called "quality of life" as a measure of health is mainly a subjective notion shaped by culture, surroundings and other psycho-social factors (Franco et al. 2009; Richter et al. 2008; Steptoe et al. 2011).

The above pragmatic definition of health as a state of having adequate physical and mental independence in activities of daily living requires identifying a set of measurable parameters at the most fundamental level of biological organization. Analyzing the components of the homeodynamic space can be an objective way to quantify health at the level of cells, tissues and the body. The following three lines of investigation may be useful to develop evidence-based markers of health:

1. Establishing immediate and delayed stress response profiles of cells, organisms and humans at different ages (Demirovic and Rattan 2013). This will also include developing methods for measuring resilience and robustness, in terms of biological and psychological parameters of well-ness and well-being, respectively (Kitano 2007; Kriete 2013).

2. Developing methods for measuring the limits of health at the individual level. Relationship between stress tolerance, recovery, survival, innate immune response and longevity could be studied at different levels and ages, using a variety of systems and making use of the most comprehensive longitudinal studies examining health-related genetic and non-genetic processes (Yashin et al. 2013). Furthermore, converting multidimensional information about the number of determinants of health at different levels to a single number expressing the biological age or the frailty status or the size of the homeodynamic space could be achieved by applying bioinformatics and mathematical modelling (Budovsky et al. 2007; Mitnitski et al. 2007; Mitnitski et al. 2013; Tacutu et al. 2010).

3. Developing physiological and psychological criteria and methods to monitor health improvement (as measured by health markers discussed above) by physical, nutritional or other interventions, including hormesis (Rattan 2012a; Rattan 2004; Rattan 2008a; Rattan 2008c; Rattan and Demirovic 2009).

\section{The rationale and the perspective}

The scientific rationale for the need of understanding and defining health is the present crisis in dealing with lifestyle diseases. The traditional biomedical approach of disease-directed treatments has now come face-toface with the big challenge of how to deal with physical, mental and social health issues where there are no clearcut causative agents or "enemies" (such as germs, bacteria, viruses, pollutants etc.). With increased life expectancy and rapidly changing demographic 
structures, major conditions such as ageing, metabolic disorders, depression, dementia, osteoporosis, sarcopenia, incontinence, malnutrition, and several types of cancers are known to be due to the generalised processes of life and their interactive networks.

Another important socio-political reason for the need of having such health-oriented thoughts, in contrast to the disease-oriented views, is the realisation that continuing the disease-oriented approach to health is scientifically and socio-economic-politically not compatible with the highly desirable future of global health and sustainability. Several prospective analyses have shown that the prevalent disease-management or disease-treatment approaches are economically, socially and psychologically unsustainable as compared with health-oriented and preventive strategies (Carnes 2011; Carnes et al. 2012; Olshansky et al. 2011).

Although various ongoing discourses on health catch on to some reality, it is important to find out how these various discourses may be interrelated as regards their interests, concerns, problems and needs (Faragher 2009; Fulop et al. 2010; Gems and Partridge 2013; Lopez-Otin et al. 2013; Mitnitski et al. 2007; Phelan et al. 2004). That would further clarify as to how health is an important issue and how we can do something about it. We cannot continue with the "more of the same approach" in matters of individual, social and public health.

Acknowledgments Laboratory of Cellular Ageing is financially partially supported by a research Grant from LVMH Recherche, Saint Jean de Braye, France.

\section{References}

Blagosklonny MV (2012) Cell cycle arrest is not yet senescence, which is not just cell cycle arrest: terminology for TORdriven aging. Aging (Albany NY) 4:159-165

Budovsky A, Abramovich A, Cohen RM, Chalifa-Caspi V, Fraifeld VE (2007) Longevity network: construction and implications. Mech Ageing Dev 128:117-124

Calabrese EJ, Bachmann KA, Bailer AJ, Bolger PM et al (2007) Biological stress response terminology: integrating the concepts of adaptive response and preconditioning stress within a hormetic dose-response framework. Toxicol Appl Pharmacol 222:122-128

Carnes BA (2011) What is lifespan regulation and why does it exist? Biogerontology 12:367-374

Carnes BA, Olshansky SJ, Hayflick L (2012) Can human biology allow most of us to become centenarians? J Gerontol A Biol Sci Med Sci 68(2):136-142
Contrada RJ, Baum A (eds) (2011) Handbook of stress science: psychology, biology, and health. Springer, New York

Demirovic D, Rattan SI (2013) Establishing cellular stress response profiles as biomarkers of homeodynamics, health and hormesis. Exp Gerontol 48:94-98

Faragher RG (2009) What could advances in the biology of ageing mean for the quality of later life? Qual Ageing 10:30-38

Farrelly C (2012) 'Positive biology' as a new paradigm for the medical sciences. Focusing on people who live long, happy, healthy lives might hold the key to improving human well-being. EMBO Rep 13:186-188

Franceschi C, Valensin S, Bonafè M, Paolisso G, Yashin AI, Monti D, De Benedictis G (2000) The network and the remodeling theories of aging: historical background and new perspectives. Exp Gerontol 35:879-896

Franco OH, Karnik K, Osborne G, Ordovas JM, Catt M, van der Ouderaa F (2009) Changing course in ageing research: the healthy ageing phenotype. Maturitas 63:13-19

Fulop T, Larbi A, Witkowski JM, McElhaney J, Loeb M, Mitnitski A, Pawelec G (2010) Aging, frailty and age-related diseases. Biogerontology 11:547-563

Gems D, Partridge L (2013) Genetics of longevity in model organisms: debates and paradigm shifts. Annu Rev Physiol 75:621-644

Holliday R (2007) Ageing: the paradox of life. Springer, Dordrecht

Holliday R (2009) The extreme arrogance of anti-aging medicine. Biogerontology 10:223-228

Hubbard RE, Woodhouse KW (2010) Frailty, inflammation and the elderly. Biogerontology 11:635-641

Kitano H (2007) Towards a theory of biological robustness. Mol Syst Biol 3:137

Kriete A (2013) Robustness and aging- a systems-level perspective. Biosystems 112:37-48

Le Bourg E (2013) Obsolete ideas and logical confusions can be obstacles for biogerontology research. Biogerontology 14:221-227

Lopez-Otin C, Blasco MA, Partridge L, Serrano M, Kroemer G (2013) The hallmarks of aging. Cell 153:1194-1217

Mitnitski A, Song X, Rockwood K (2007) Improvement and decline in health status from late middle age: modeling age-related changes in deficit accumulation. Exp Gerontol 42:1109-1115

Mitnitski A, Song X, Rockwood K (2013) Assessing biological aging: the origin of deficit accumulation. Biogerontology 14. doi:10.1007/s10522-013-9446-3

Montesanto A, Lagani V, Martino C, Dato S et al (2010) A novel, population-specific approach to define frailty. Age 32:385-395

Olshansky SJ, Biggs S, Achenbaum WA, Davison GC et al (2011) The global agenda council on the ageing society: policy principles. Global Policy 2:97-105

Phelan EA, Anderson LA, LaCroix AZ, Larson EB (2004) Older adults' views of "successful aging"-how do they compare with researchers' definitions? J Am Geriatr Soc 52:211-216

Rattan SIS (2004) Aging, anti-aging, and hormesis. Mech Ageing Dev 125:285-289

Rattan SIS (2006) Theories of biological aging: genes, proteins and free radicals. Free Radic Res 40:1230-1238 
Rattan SIS (2007a) Homeostasis, homeodynamics, and aging. In: Birren J (ed) Encyclopedia of gerontology. Elsevier, Oxford, pp 696-699

Rattan SIS (2007b) The science of healthy aging: genes, milieu, and chance. Ann NY Acad Sci 1114:1-10

Rattan SIS (2008a) Hormesis in aging. Ageing Res Rev 7:63-78

Rattan SIS (2008b) Increased molecular damage and heterogeneity as the basis of aging. Biol Chem 389:267-272

Rattan SIS (2008c) Principles and practice of hormetic treatment of aging and age-related diseases. Hum Exp Toxicol 27:151-157

Rattan SI (2012a) Rationale and methods of discovering hormetins as drugs for healthy ageing. Expert Opin Drug Discov 7:439-448

Rattan SIS (2012b) Biogerontology: from here to where? The Lord Cohen Medal Lecture-2011. Biogerontology 13: 83-91

Rattan SIS, Demirovic D (2009) Hormesis and aging. In: Mattson MP, Calabrese E (eds) Hormesis: a revolution in biology, toxicology and medicine. Springer, New York, pp 153-175
Richter J, Schwarz M, Bauer B (2008) Personality characteristics determine health-related quality of life as an outcome indicator of geriatric inpatient rehabilitation. Curr Gerontol Geriatr Res. doi:10.1155/2008/474618:8 Article ID 474618

Shelton JD (2013) Ensuring health in universal health coverage. Nature 493:453

Steptoe A, Leigh ES, Kumari M (2011) Positive affect and distressed affect over the day in older people. Psychol Aging 26:956-965

Tacutu R, Budovsky A, Fraifeld VE (2010) The NetAge database: a compendium of networks for longevity, age-related diseases and associated processes. Biogerontology 11:513-522

Yashin AI, Arbeev KG, Wu D, Arbeeva LS, Kulminski A, Akushevich I, Culminskaya I, Stallard E, Ukraintseva SV (2013) How lifespan associated genes modulate aging changes: lessons from analysis of longitudinal data. Front Genet $4: 3$

Yates FE (1994) Order and complexity in dynamical systems: homeodynamics as a generalized mechanics for biology. Math Comput Model 19:49-74 